\title{
Frontières
}

\section{Être ou avoir, voilà la tension...}

Daniel Laguitton

Volume 12, numéro 1, automne 1999

URI : https://id.erudit.org/iderudit/1074520ar

DOI : https://doi.org/10.7202/1074520ar

Aller au sommaire du numéro

Éditeur(s)

Université du Québec à Montréal

ISSN

1180-3479 (imprimé)

1916-0976 (numérique)

Découvrir la revue

Citer ce document

Laguitton, D. (1999). Être ou avoir, voilà la tension... Frontières, 12(1), 111-114.

https://doi.org/10.7202/1074520ar d'utilisation que vous pouvez consulter en ligne.

https://apropos.erudit.org/fr/usagers/politique-dutilisation/ 


\section{Être ou avoir, voilà la tension...}

par Daniel Laguitton, écrivain-éducateur.

La tension existentielle qui écartèle l'homme entre les exigences de l'avoir et celles de l'être est une différence de potentiel porteuse d'espoir ou de calamité. On s'électrocute aux bornes d'une prise électrique quand on ne sait pas s'en servir, on éclaire la nuit la plus sombre lorsque l'on sait en chevaucher les pôles.

\section{PRISON D'AVOIR}

Au pôle de l'avoir règne la volonté, au pôle de l'être la bonne volonté, disposition du coeur, alors que la première est un attribut du "petit-moi», cet intendant avare qui compare, désire, préfère, et fait de l'univers un «autre», un problème à résoudre, ou un royaume à dominer. Le désir insatiable du petit-moi paresseux est de trouver un raccourci existentiel, une fausse identité qui l'exempterait des exigences de l'être. Pour ce faire il découpe de son scalpel une région de l'univers à laquelle il s'identifie, réalisant ainsi une rupture cosmique et ontologique symbolisée dans la tradition biblique par la perte $d u$ jardin originel: l'univers est amputé de moi, je suis amputé de lui. Un cri jaillit alors des profondeurs et le doigt d'Adam s'éloigne de celui de Dieu sur la célèbre fresque de Michel-Ange. Plus j'ai (ou plus je veux avoir), moins je suis, louvoiement perpétuel de ma transhumance, pesanteur mortifère ou force motrice de salut.

En deçà des inventaires de greniers et de garages encombrés, l'avoir commence par le nom. Tagore, poète et mystique de l'Inde, décrit de façon poignante l'univers carcéral du nom, cette cellule d'avoir que l'on peut appeler «égonymique» dans la mesure où le nom est à l'ego ce que le toponyme est au lieu:

Mon propre nom est une prison

où je m'enferme et je pleure.

Sans cesse je m'occupe à en élever tout autour de moi la paroi; et tandis que, de jour en jour, cette paroi grandit vers le ciel, dans l'obscurité de son ombre, je perds de vue mon être véritable ${ }^{1}$.

Là où règne l'avoir règne aussi l'orgueil dont la pire forme serait, selon Flaubert, «de se mépriser soi-même», ce qui indique que la soif d'être ne s'abreuve pas dans un mépris de l'avoir. Maître Eckhart est particulièrement explicite à ce sujet lorsque dans ses sermons il rabroue ceux qui font de la mortification un commerce spirituel et qu'il compare à «des ânes qui n'ont rien compris». Plus près de nous, Gabriel Marcel ${ }^{2}$ souligne le piège d'une «concupiscence de la sincérité, qui n'est que l'exaltation de toutes les forces de négation qui sont en moi et qui peut-être la forme la plus proprement satanique du suicide: l'extrême orgueil, par une perversion sans mesure, y simule l'extrême humilité». Martin Buber ${ }^{3}$ écrit quant à lui: "Entreprise folle et désespérée, que de s'écarter du chemin de sa propre vie pour chercher Dieu! Se fut-on approprié toute la sagesse de la solitude et toute la vertu du recueillement, on manquerait à trouver Dieu».

\section{JE VEUX AVOIR, DONC JE SUIS ?}

Quelques siècles se sont écoulés depuis le retentissant «je pense donc je suis» et le déferlement rationaliste qui l'a suivi. Si l'on en juge par le bilan de quatre siècles de raison triomphante, le royaume de la pensée se superpose à s'y méprendre à celui de l'avoir et à la carence d'être qui le caractérise. Le règne de l'avoir se manifeste en désir insatiable de gratification personnelle, le Tanha ou Trishnâ que la seconde des nobles vérités du bouddhisme identifie comme cause principale du déséquilibre (Duhka) de l'être humain. Ne serait-il pas plus clair, compte tenu de l'usage prédateur qui est généralement fait de la pensée, de reformuler le cogito en explicitant la séquence égocentrique qui l'anime: je pense, donc je veux saisir; je veux saisir, donc je veux avoir; je veux avoir, donc je suis? La préséance de l'être s'affirme-t-elle par la capacité que nous avons de nous en éloigner? Nous disons couramment aujourd'hui "avoir» un certain âge, "avoir» une famille, une nationalité, des aptitudes, un métier, un conjoint, des enfants, des biens, un lot au cimetière, une épitaphe, une réputation, un destin. Il existe pourtant des expressions équivalentes utilisant le verbe être (être d'un certain âge, être membre de telle famille ou de telle nationalité, être doué, être boulanger, marié, père de famille, riche, décédé, célèbre, etc.), mais l'avoir gagne partout $\mathrm{du}$ terrain. Pour illustrer la progression insidieuse de l'avoir aux dépens de l'être, Éric Fromm ${ }^{4}$, grand observateur de la psyché, souligne une tendance accélérée à substituer les noms aux verbes, ce qui dénote un glissement vers le règne de l'avoir. «Voici un exemple typique, écritil, quoique légèrement exagéré, du langage d'aujourd'hui. Supposons qu'une personne qui sollicite l'aide d'un psychanalyste engage ainsi la conversation: Docteur, j'ai un problème, j'ai des insomnies. Bien que j'aie une belle maison, de beaux enfants et une union heureuse, j'ai bien des soucis. Il y a quelques décennies, au lieu de j'ai bien des problèmes, le patient aurait probablement dit je suis perplexe, au lieu de j'ai des insomnies, je ne peux pas dormir, au lieu de j'ai une union heureuse, je suis heureux en ménage. Ceux qui croient qu'avoir est l'une des catégories les plus naturelles de l'existence humaine, poursuit Fromm, seraient très surpris d'apprendre que de nombreux langages n'ont aucun mot correspondant à "avoir". Le mot correspondant à "avoir" évolue en relation avec le développement de la propriété privée alors qu'il est absent dans les sociétés où prédomine la propriété fonctionnelle, c'est-à-dire la possession pour l'usage».

Évoquant le mouvement relationnel inhérent à l'être, Martin Buber écrit que 
«les bases du langage ne sont pas des noms de choses mais des rapports». Lorsque le prologue de l'Évangile de Jean affirme: "Au commencement était le Verbe», il désigne avant tout un rapport originel qu'il nomme Logos, parole vivante qui ne doit pas être confondue avec la photographie mentale du nométiquette, l'onoma d'onomatopée que le dictionnaire définit comme «le nom, par opposition à la chose nommée». Les tout premiers mots du Daodejing 5 font la même distinction entre la Voie vivante et la Voie nommée:

La Voie dont on parle

n'est pas la Voie suprême;

concepts et noms

sont des illusions.

Toute réalité

émane du sans-nom;

le nom est ce qui divise

en donnant à toute chose

une identité.

La domination du pôle «avoir» est avant tout fondée sur un pathétique projet d'immortalité appelé ego en latin comme en grec et dont on a dit qu'il est un «mensonge vital». Le caractère, affirme Ernest Becker6, "est la façade que l'on présente au monde et qui cache une défaite intérieure.» Et l'anthropologue d'ajouter: "Tout développement de notre personnage revient à cacher la masse de tissus scarifiés qui palpite dans nos rêves.» «La plupart d'entre nous, dès la sortie de l'enfance, avons réprimé notre vision $\mathrm{du}$ miracle fondamental de la création.» Exilés dans l'avoir, beaucoup passent l'essentiel de leur existence à anesthésier leur nostalgie du pays natal de l'être dont ils ont une mémoire diffuse et à soigner les angoisses de leur ego algophobe. Éric Fromm décrit le «consommateur» moderne comme «un éternel nourrisson pleurant pour avoir son biberon.» Cela est évident, dit-il, dans les phénomènes pathologiques comme l'alcoolisme et les drogues. «Apparemment, si nous classons à part ces deux modes de dépendance, c'est que leurs effets compromettent les obligations sociales de ceux qui en sont victimes.» Un répertoire des substances et comportements qui compromettent l'épanouissement ontologiques des prisonniers de l'avoir serait autrement plus volumineux! L'habitude est un triomphe de l'avoir (le mot vient du latin habere) et, au lieu de dire "avoir l'habitude», on devrait dire «se faire avoir par l'habitude».

Selon Gabriel Marcel, «l'opposition entre le désir et l'amour constitue une illustration très importante de l'opposition entre l'avoir et l'être.» «Désirer, en effet, écrit-il, c'est avoir en n'ayant pas.
La symétrie est d'ailleurs absolue entre la convoitise et l'angoisse que j'éprouve à l'idée que je vais perdre ce que j'ai, ce que je croyais avoir, ce que je n'ai déjà plus.» Lao-Tseu l'avait affirmé vingt-cinq siècles plus tôt:

La réussite produit autant de peur que l'échec;

l'adversité est à la mesure de l'ego.

Pourquoi réussite et échec sontils équivalents?

Parce que la peur de perdre ce que l'on a gagné

est identique à celle de ne pas

retrouver ce qu'on a perdu.

Pourquoi l'adversité est-elle à la

mesure de l'ego?

Parce que l'ego en est la cause.

Sans ego, comment pourrais-je

percevoir l'adversité?

«Avoir, assurément, poursuit Gabriel Marcel, cela peut signifier, et cela signifie même en principe: avoir à soi, garder pour soi, dissimuler. Ici l'exemple le plus intéressant, le plus typique, c'est avoir un secret. [...] Cette possibilité de trahison ou de découverte lui est inhérente et contribue à le définir en tant que secret. Ceci n'est pas unique, mais se vérifie partout où nous sommes en présence de l'avoir au sens fort. La caractéristique de l'avoir, c'est d'être exposable.» La relation entre l'avoir et le secret n'est sans doute nulle part plus évidente que dans le secret bancaire et dans la pudeur dont nous entourons généralement nos rapports avec l'argent. L'expression «avoir honte» devient presque un pléonasme dans la mesure où «avoir» et «dissimulation» vont de pair. Plus l'avoir nous mobilise, plus il nous confronte à notre finitude et plus il attise notre soif d'infini. «Nos possessions nous dévorent,» constate encore Gabriel Marcel, «ceci est d'autant plus vrai, chose étrange, que nous sommes plus inertes en face d'objets en eux-mêmes inertes, et d'autant plus faux que nous sommes plus vitalement, plus activement liés à quelque chose qui serait comme la matière même, la matière perpétuellement renouvelée d'une création personnelle (que ce soit le jardin de celui qui le cultive, la ferme de celui qui l'exploite, le piano ou le violon du musicien, le laboratoire du savant).» Au sujet de l'inertie caractéristique d'un mode de vie centré sur l'avoir, Éric Fromm constatait: «En ce qui concerne le temps consacré aux loisirs, l'automobile, la télévision, les voyages et le sexe sont les principaux objets de la consommation actuelle et au lieu de parler à leur propos "d'activités de loisir"., on ferait beaucoup mieux de les appeler "passivités de loisir"». Boulimique insatiable, l'homme d'avoir tente d'apaiser la faim et sa soif d'être et une des ironies de son assujettissement à l'avoir serait illustrée par les statistiques décrivant l'expansion rapide des ventes de cadenas et autres systèmes de protection de la propriété privée, ventes dont on peut parier qu'elles s'accompagnent d'une croissance proportionnelle de la consommation de produits laxatifs! Matérialisme et constipation seraient-ils en corrélation très étroite? Avoir, c'est en effet non seulement désirer et consommer, mais c'est surtout retenir.

\section{LÂCHER PRISE}

Toutes les traditions spirituelles offrent à l'humanité exilée dans l'avoir l'espoir d'un retour vers l'être. Éric Fromm a cette phrase encourageante: "Je crois qu'un très grand nombre de groupes et d'individus se dirigent vers le mode être, qu'ils représentent une nouvelle tendance dépassant l'orientation avoir de la majorité et qu'ils ont une signification historique». La mouvance, fut-elle en masse, commence au coeur de chaque itinérant. Dans le mythe grec, Orphée obtient la promesse qu'Euridyce sera libérée du monde des ténèbres et le suivra à condition qu'il ne se retourne pas en chemin. Succombant au doute, il faillit à sa promesse et la nymphe qu'il aime est reprise par l'ombre. Dans le Livre de la libération par l'écoute, mieux connu sous le nom de Livre tibétain des morts, le défunt est exhorté à ne pas se laisser distraire par les créations de son mental: «Va, noble fils de lumière, va ton chemin sans te retourner». Pour qui s'efforce de répondre à l'appel de l'être, il est bien difficile de ne pas se retourner vers l'avoir. "Ne nous laissez pas succomber à la tentation...», supplique de pèlerin entre l'avoir et l'être.

Un article récent du magazine Nouvelles Clés relatait un échange public entre un homme affirmant avoir fait l'expérience de "l'être pur» par extinction du petit-moi et un théologien qui exprimait ses doutes quant à l'authenticité de cette interprétation. Selon ce dernier, il s'agissait tout au plus d'un retour aux racines du Soi, sentiment d'exister en deçà de toute différenciation et de toute rationalisation $\mathrm{du}$ monde. Cette expérience du «Soi immanent»se distinguerait, selon le docte critique, de celle $\mathrm{du}$ «divin transcendant» dont parlent les mystiques. Enseigne-t-il à ses heures, ce divin cartographe, que l'Être qu'il nomme Dieu est partout, y compris aux racines du Soi? Et pour ne pas se contredire ici, il faut bien admettre que 
si le mouvement vers l'avoir est un éloignement par rapport à l'être, l'être est aussi dans l'avoir. "Les voies de l'excès mènent au palais de la sagesse», affirmait William Blake. Les chevaliers d'avoir sont de temps à autre désarçonnés sur leur chemin de Damas. S'il s'enfourche comme une Rossinante, l'avoir est aussi un Cheval de Troie qui se pénètre au sens biblique: si nos possessions nous dévorent, elles peuvent, comme la baleine de Jonas, être également des outils de connaissance, des véhicules de transformation. Ce n'est pas une excuse pour se vautrer plus complaisamment encore dans l'avoir.

\section{AVOIR TOUT À PERDRE}

"Le siège de la souffrance semble bien être la zone où l'avoir débouche dans l'être» affirme Gabriel Marcel. Ce passage de l'avoir à l'être est manifesté dans le don, au sujet duquel Éric Fromm reprend une analogie attribuée à Max Hunziger: "Nous disons que ce verre est bleu précisément parce qu'il ne retient pas les ondes bleues. Il est qualifié non par ce qu'il possède, mais par ce qu'il donne». Les exemples abondent pour illustrer l'étroite relation qui fait du don une passerelle vers la cité de l'être. «Une des pires souffrances de la misère, pour qui n'est pas incapable d'amour, écrit Gide dans son Journal, c'est de devoir toujours recevoir, de ne pouvoir jamais donner.» «C'est quand on a tout donné, quand on ne tient plus à rien qu'on possède tout» affirme Marcel Jouhandeau dans Réflexions sur la vieillesse et la mort alors que, pour Gilbert Cesbron, «Cette pièce n'est à toi que si elle quitte ta main.» La voix posthume de Janis Joplin égraine encore fréquemment à la radio le refrain d'une chanson célèbre de Kris Kristopherson, Freedom is nothing left to lose - la liberté, c'est de n'avoir plus rien à perdre, formule séduisante qui devient plus pertinente si l'on se réfère aux racines du verbe "perdre»du latin per-dare (donner complètement). La liberté suprême commencerait alors dans le fait d'avoir tout à perdre pour culminer dans celui d'avoir tout donné. "Père, entre tes mains je remets mon esprit», don sans retour, passerelle lumineuse vers l'être. La liberté qu'on a, celle du libre-choix, devient la liberté qu'on est, par le don assumé.

Avoir pour donner, avoir pour perdre, avoir pour pardonner (per-donare est synonyme de per-dare, donner entièrement, remettre), avoir pour abandonner: "lâcher prise», dit-on aujourd'hui. Plus facile à dire qu'à faire. Apprendre à mourir, c'est apprendre à être: les expériences de mort imminente physique ou psychique et la transformation personnelle qui les accompagne généralement chez ceux qui les ont vécues sont là pour nous le rappeler. Le poète anglo-américain T. S. Eliot ${ }^{7}$, dont la tétralogie Four Quartets constitue un véritable journal de voyage d'explorateur de l'Être, évoque en ces mots la dissolution du filet de l'avoir:

Pas un geste, ai-je enjoint à mon

âme,

Que le noir t'enveloppe,

Il est la nuit de Dieu.

Comme un théâtre éteint pour

changer de décor

En un grondement sourd qui

monte des coulisses,

En un repliement de la nuit sur elle-même,

On sait qu'alors s'en vont col-

lines et forêts,

Panoramas lointains, façades

imposantes;

Ou comme en son tunnel, entre

deux stations

Quand la rame de métro fait un

arrêt trop long

Un lourd silence étreint toute

conversation

Et un vide mental s'inscrit sur les visages

N'y laissant bientôt plus qu'une terreur perplexe;

Ou comme sous l'éther l'esprit

reste conscient

Mais n'est conscient de rien -

Pas un geste, ai-je enjoint à mon

âme,

Attends sans espoir car tout

espoir se tromperait d'objet;

Attends sans amour, car tout

amour se tromperait d'amant;

Reste la foi, mais comme l'espoir

et comme l'amour

Elle consiste à attendre,

Attendre sans penser, car on n'y est pas prêt:

La nuit sera lumière, le repos

sera danse.

Murmure de torrents, éclairs en

hiver.

Le thym discret et la fraise sau-

vage,

Les rires dans le jardin, sont

l'écho d'un délice

Accessible en tout temps mais qui par contre exige,

Implique, l'agonie de mourir et de naître.

\section{ATTENTE ACTIVE}

Souffrir l'attente active est le travail de parturition qui nous échoit dans l'antichambre de l'être. «Le véritable travail est de savoir attendre», écrivait Jean Rostand. «Ce n'est que dans le processus d'une relation mutuelle vivante que l'autre et moi-même pouvons franchir la barrière qui nous sépare, dans la mesure où nous participons à la même danse de la vie», affirme aussi Éric Fromm qui ajoute "Vivre bien, ce n'est plus seulement satisfaire une exigence éthique ou religieuse. Pour la première fois dans l'histoire, la survie physique de la race humaine dépend d'un changement radical du coeur humain. Mais ce changement n'est possible que dans la mesure où interviennent des changements économiques et sociaux rigoureux capables de donner au coeur humain la chance de changer et le courage et l'envie d'accomplir ce changement». Selon Martin Buber, le changement exigé est l'ouverture à la relation: "Un délai est imparti au fils d'homme pour échanger contre un lien spirituel, c'est-à-dire contre une relation, le lien naturel qui l'unissait à l'univers.»

Dès que le mot «changement» est prononcé, les réformateurs de tout poil se lèvent, ingénieurs, idéologues, mercenaires. Mais Fromm les met en garde contre les idéaux trompeurs: "L'idée d'une société sans classes, dans un monde prétendument socialiste imprégné de l'esprit de cupidité est aussi illusoire - et dangereuse - que l'idée d'une paix permanente entre nations cupides.» Le psychanalyste prend plutôt position à mi-chemin entre ceux qui pensent que l'on doit commencer par changer radicalement la structure politique et économique pour induire le changement de l'esprit humain et «ceux qui soutiennent que c'est d'abord la nature des être humains qui doit changer - leur état d'esprit, leurs valeurs, leur caractère- et que ce n'est qu'alors qu'une société vraiment humaine pourra être construite.» "Ils ne voient pas», écrit-il au sujet des premiers, «que la nouvelle élite, étant motivée par le même caractère que l'ancienne, aura tendance à reproduire les conditions de l'ancienne société dans le cadre de nouvelles institutions sociopolitiques créées par la révolution». Au sujet des seconds, son pronostic n'est pas plus favorable: "L'histoire de la race humaine leur donne tort, le changement purement psychique a toujours été limité à des sphères privées et à de petites oasis, ou bien il a été totalement inefficace quand la prédication des valeurs spirituelles se combinait avec la pratique de valeurs opposées.»

Bien qu'il esquisse certains changements institutionnels concrets, selon lui souhaitables, pour que la transformation 
des individus aille de pair avec un changement structurel social, nous ne retiendrons ici que la nécessité et l'orientation générale des changements souhaités par Éric Fromm. Cette orientation est manifeste dans le rapprochement qu'il fait de l'oeuvre d'Albert Schweitzer, le théologien protestant, et de Maître Eckhart, le prédicateur dominicain: "La tâche de l'homme n'est pas de se retirer dans l'ambiance de son égotisme spirituel, ni de se détacher des affaires de ce monde, mais de mener une vie active où chacun essaie de contribuer pour sa part à la perfection spirituelle de la société.» Citant l'auteur de Small is beautiful, l'économiste E.F. Schumacher, Fromm affirme aussi que «Nos échecs sont la conséquence de nos succès et que nos techniques doivent être subordonnées à nos véritables besoins humains". «L'économie en tant que principe de vie écrit en effet Schumacher, est une maladie mortelle.» «Tous ses grands Maîtres ont dit à l'humanité que l'économie ne doit pas être principe de vie; il est aujourd'hui évident qu'elle ne peut pas l'être. Si l'on veut dépeindre plus en détail la maladie mortelle, on peut dire qu'elle est comparable à l'alcoolisme ou à la drogue. Il importe peu que cet esclavage apparaisse sous une forme plus égoïste ou plus altruiste, qu'il cherche sa satisfaction d'une façon vulgairement matérialiste ou bien d'une façon artistiquement, culturellement ou scientifiquement raffinée. Le poison est poison, même s'il est enveloppé dans un papier d'argent.» Schumacher de poursuivre: «Si la culture spirituelle, la culture de l'homme intérieur est négligée, l'égoïsme, alors, demeure chez l'homme le pouvoir dominant, et un système ou prévaut l'égoïsme, comme l'est le capitalisme, convient mieux à cette orientation qu'un système où prévaut l'amour du prochain».

En pragmatiste qu'il était, Éric Fromm conclut: «Je ne pense pas que rien de durable puisse être obtenu par des individus qui souffrent d'un malaise général et pour lesquels un changement de caractère est nécessaire, à moins qu'ils ne changent leur mode de vie en accord avec le changement de caractère qu'ils veulent réaliser». Concrètement parlant, l'alcoolique doit cesser de fréquenter les bars, l'ergomane d'aligner les semaines de soixante-dix heures, et l'avare de thésauriser, pour ne mentionner que ces trois caricatures du revirement nécessaire.

Prendre conscience d'un problème et en connaître la solution est une chose, mettre la solution en pratique en est une autre. On doit à l'auteur de Vol de nuit une formule destinée aux consommateurs de solutions toutes faites: "Dans la vie, il n'y a pas de solutions. Il y a des forces en marche: il faut les créer, et les solutions suivent». Créer ces forces exige d'abord d'être en possession de tous ses moyens en s'éveillant de l'hypnose matérialiste. "Nous ne sommes pas en pleine possession de nos esprits, écrit Fromm à ce sujet, parce que des méthodes hypnoïdes sont employées pour nous endoctriner». Et si nous sombrons dans une léthargie toujours plus profonde, c'est sans doute que nous savons inconsciemment que la souffrance est incontournable pour qui s'éveille à l'être: «La suppression du poison de la suggestion massive aura sur les consommateurs un effet à peine différent des symptômes de manque que connaissent les drogués quand ils cessent de prendre des drogues», prévient le psychanalyste. Le «bas-fond» incontournable que prépare la consommation à outrance est toutefois, comme toute crise, un mal potentiellement salutaire: nous tirerons leçon des errances passées ou nous périrons dans un cloaque d'avoir.

Dans la Bible traduite par André Chouraki, l'expression «en marche» est utilisée là où les traductions traditionnelles utilisent «bienheureux», mot dont les racines évoquent également la mouvance, mais que l'usage a recouvert d'une patine édulcorée. Cette marche est alimentée par la tension mentionnée plus haut entre l'avoir et l'être. Pour reprendre l'analogie proposée en préambule, l'avoir correspondrait à la borne «excitée» d'un circuit électrique, l'être à la borne neutre, à la terre. Mû par (et ému vers) l'avoir, l'homme en proie au désir ou à l'aversion se comporte en Sisyphe roulant son fardeau à contrecourant de l'être. En mouvement vers l'être, il s'écoule dans l'océan primordial. L'auteur des Four Quartets exhorte les voyageurs de cette transhumance à ne pas s'arrêter en route:

En avant, voyageurs! ne pensez

pas à fuir

Vers un autre présent ou vers un avenir;

Vous n'êtes plus les gens qui ont quitté la gare

$\mathrm{Ni}$ ceux qui atteindront quelque destination,

Les rails déjà franchis se joignent

à l'horizon

Et sur le pont vibrant du vais-

seau ferroviaire

Une brèche s'étend en arrière de vous,

Ne dites pas ces mots «le passé est fini»
Ou «l'avenir s'en vient».

En avant, compagnons, qui pensez voyager,

Vous n'êtes plus les gens qui ont appareillé

$\mathrm{Ni}$ ceux qui dès demain descendront du navire.

Ici, entre deux rives, le temps n'existe plus,

L'avenir à vos yeux se fond dans le passé.

Dans l'agir sans action vous entendrez ceci:

«Quel que soit son mobile à

l'instant de sa mort»

(Et la mort est à chaque instant)

- Celui qui meurt nourrit ainsi la

vie des autres:

Sans penser au fruit de l'action, en avant.

Pour souligner l'éternel recommencement de cette marche entre deux pôles, Eliot d'ajouter ce qui sera notre dernière citation:

Nous continuerons le voyage

Et au bout de l'exploration

Touchant l'originel rivage

D'un savoir neuf, le connâ̂trons...

\section{Notes}

1 Rabindranath TAGORE, L'offrande Lyri$q u e$, traduction André Gide, Gallimard.

2 Les citations de Gabriel MARCEL sont tirées de Être et avoir, Paris, éditions Montaigne, 1968.

3 Les citations de Martin BUBER sont tirées de Je et Tu, Paris, Aubier-Montaigne, 1969.

4 Les citations de Éric Fromm sont tirées de Avoir ou être, traduction Théo CARLIER, Paris, Robert Laffont, 1978.

5 Les citations de Lao-Tseu sont extraites de La voie du coeur selon un sage, une interprétation du Tao Te King de LAO-TSEU, D. LAGUITTON, 1993, Diffusion Raffin Montréal (voir www.granby.net/ d_lag).

6 Les citations de Ernest BECKER sont tirées de The Denial of Death, New York, Free Press, 1973.

7 Les citations de T. S. Eliot sont tirées de Entre deux rives avec Thomas Eliot, traduction de Four Quartets de T. S. Eliot avec commentaire par D. LAGUITTON (recherche éditeur). 